\title{
Symptomatic bronchogenic cyst in a six-month-old infant: Case report and review of the literature
}

Birgit Ahrens, MD, ${ }^{a}$ Joachim Wit, MD, ${ }^{b}$ Markus Schmitt, MD, ${ }^{a}$ Ulrich Wahn, MD, ${ }^{a}$ Bodo Niggemann, MD, and Karl Paul, MD, ${ }^{\text {B Berlin, Germany }}$

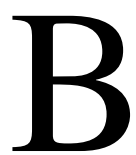

ronchogenic cysts are developmental anomalies that occur during embryogenesis. They may arise in the mediastinum or within the pulmonary parenchyma and, less frequently, below or within the diaphragm. ${ }^{1}$ Congenital cystic lesions of the lung are rare, generally benign lesions, which tend to produce few to no symptoms. However, when they present with severe and even life-threatening episodes, diagnostic and therapeutic strategy can be extremely challenging. Concerning risk of symptomatic compression, the topography of the cyst appears to be more important than the volume. ${ }^{2}$ Mediastinal masses in newborn infants with stridor are an important differential diagnosis. ${ }^{3}$

\section{Clinical Summary}

A 6-month-old infant was admitted because of increasing stridor since birth, cough, pulmonary infections, and episodes of acute respiratory distress. The baby had been hospitalized several times and extensive diagnostic work-ups had been carried out. The results were mostly unremarkable, including chest x-ray films, electrocardiograms, echocardiograms, and routine hematology and biochemistry studies. No indications of immunodeficiency, allergic diseases, or an aspiration episode were found. Despite systemic and inhaled corticosteroids, stridor and respiratory distress were persistent.

Our clinical examination revealed a eutrophic, agile infant with no acute distress at rest. However, while straining, a dominant inspiratory and expiratory stridor was noted. On auscultation, wheezing was heard, dorsal, stronger on the left than the right. The chest x-ray film on admission was unremarkable (Figure 1).

A bronchoscopic examination showed an extrinsic, nonpulsating ventral mass narrowing the tracheal lumen from the thoracic inlet to the carina and leading to a slotlike restriction in the lower third of the trachea. The tracheal diameter was reduced to approximately

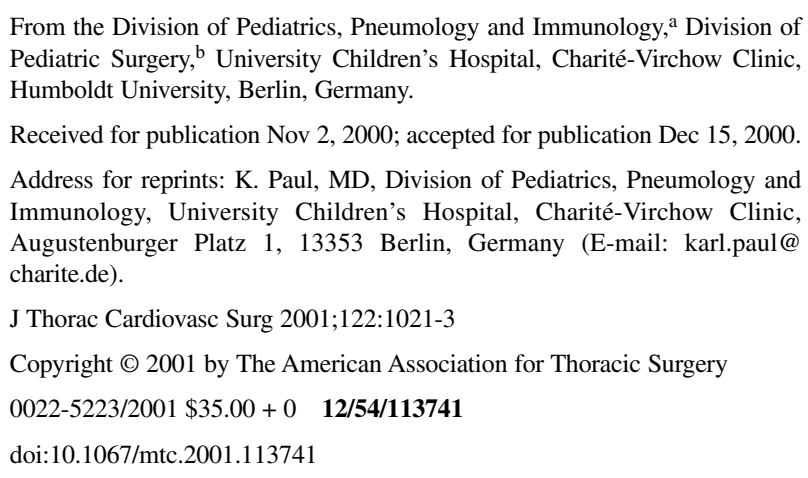

From the Division of Pediatrics, Pneumology and Immunology, ${ }^{\mathrm{a}}$ Division of Pediatric Surgery, ${ }^{\mathrm{b}}$ University Children's Hospital, Charité-Virchow Clinic, Humboldt University, Berlin, Germany.

Received for publication Nov 2, 2000; accepted for publication Dec 15, 2000.

Address for reprints: K. Paul, MD, Division of Pediatrics, Pneumology and Immunology, University Children's Hospital, Charité-Virchow Clinic, Augustenburger Platz 1, 13353 Berlin, Germany (E-mail: karl.paul@ charite.de).

J Thorac Cardiovasc Surg 2001;122:1021-3

Copyright (C) 2001 by The American Association for Thoracic Surgery $0022-5223 / 2001 \$ 35.00+0 \quad \mathbf{1 2 / 5 4} / \mathbf{1 1 3 7 4 1}$

doi: $10.1067 / \mathrm{mtc} .2001 .113741$

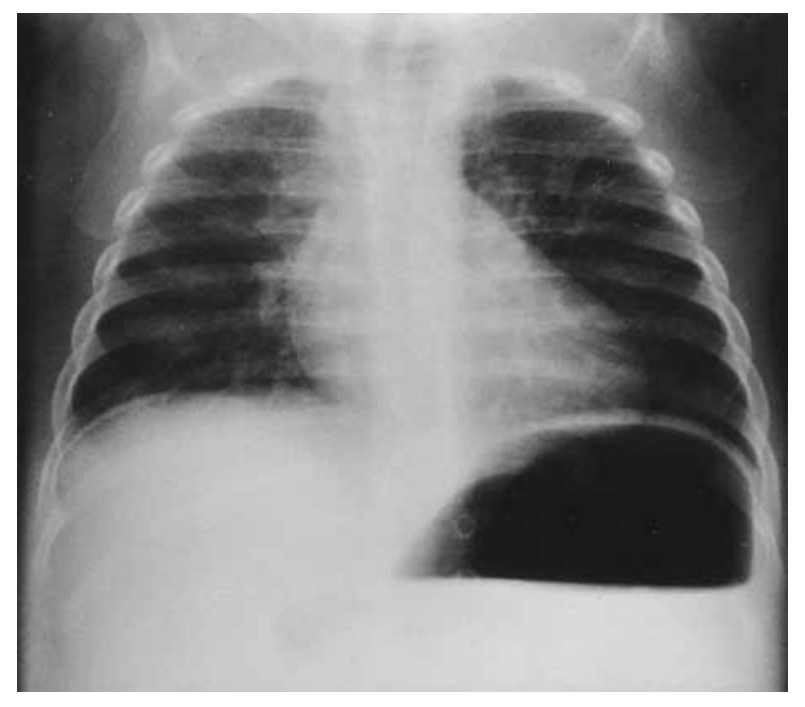

Figure 1. Chest $x$-ray film (at age 6 months) was unremarkable.

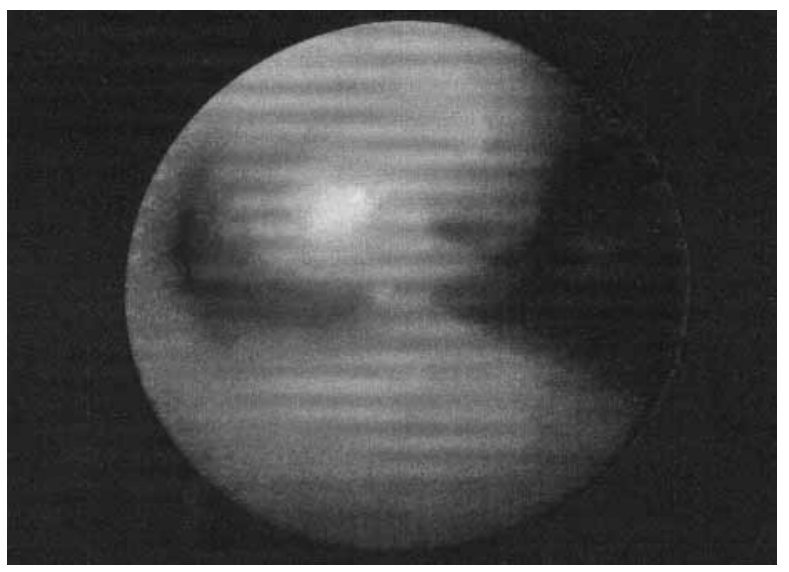

Figure 2. Preoperative bronchogram showed a slotlike restriction of the trachea (nearly $80 \%$ ).

$20 \%$ (Figure 2). An echocardiogram revealed a right-sided mediastinal structure between the superior vena cava and aorta (Figure 3). Computed tomographic scan specified size, location, and anatomic relationship of the mass: $3.5 \times 2 \times 1.5 \mathrm{~cm}$, with clear compression of the trachea and main bronchi. The structure appeared homogeneous with fluid-equivalent signal intensity (Figure 4). Results of tumor marker, tuberculin (purified protein derivative-S 10, 100), and Mycobacterium avium skin tests were unremarkable. 


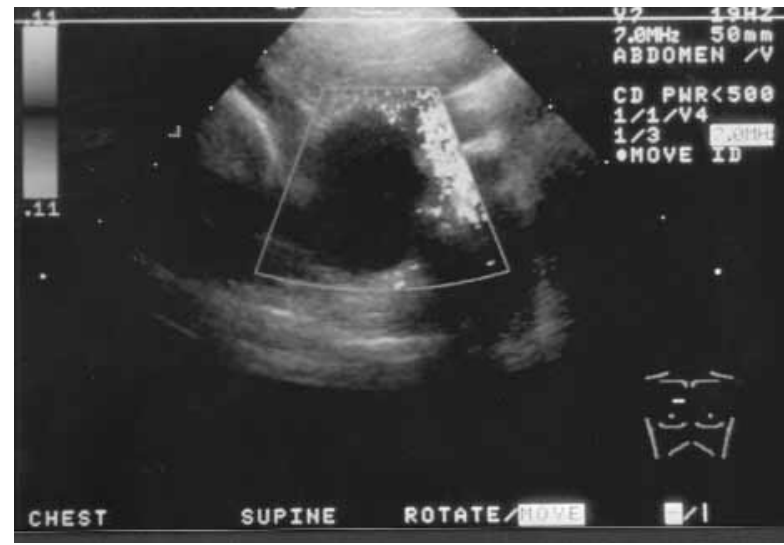

Figure 3. Echocardiogram demonstrated a right mediastinal ovoid echo-poor structure, diameter approximately $2 \mathrm{~cm}$, with no blood perfusion. Cardiac structure and function were normal.

\section{TABLE 1. Bronchogenic cysts}

\begin{tabular}{ll}
\hline Mediastinal (86\%) & Intrapulmonary (14\%) \\
\hline $\begin{array}{l}\text { Usually asymptomatic; stridor, } \\
\text { dysphagia }\end{array}$ & - Infection (75\%), dyspnea, hemoptysis \\
- Male/female = 1:1 & $\begin{array}{l}\text { Male > female; LL/UL = 2:1, usually } \\
\text { medial third }\end{array}$ \\
- Associated with spinal & - $36 \%$ may contain air \\
abnormalities & \\
- Communication with tracheal lumen & \\
possible & Location: usually on the right; posterior \\
(50\%), superior (14\%) mediastinum; \\
pericarinal (35\%)
\end{tabular}

Modified from Hahn YH. Bronchogenic cyst. CHORUS, Collaborative Hypertext of Radiology 1995. Medical College of Wisconsin, Milwaukee, Wisconsin. Retrieved March 7, 2000, from the World Wide Web: http.// chorus.rad.mcw.edu/doc/00904.html).

The finding of the mediastinal mass in combination with increasing, potentially life-threatening acute respiratory distress situations required rapid intervention. The infant underwent a thoracotomy, and a tense cystic mass was discovered and carefully removed. The mass was connected by a stalk to the right main bronchus about $1 \mathrm{~cm}$ distal to the bifurcation. Results of histologic examination were consistent with a bronchogenic cyst. A follow-up bronchogram revealed that the trachea was still asymmetric with an unstable area around the carina.

Postoperatively, the child recovered quickly and was discharged after 8 days.

\section{Discussion}

Mediastinal cysts are rare, usually asymptomatic, and often detected accidentally. Bronchogenic cysts, for instance, make up 6\% to $15 \%$ of primary mediastinal tumors. ${ }^{2}$ Mediastinal cysts result from an abnormal budding of the ventral diverticulum of the foregut or the tracheobronchial tree during embryogenesis. The respiratory budding arises between days 20 and 40 of gestation. The cleavage between the respiratory tract and the digestive tube occurs on day

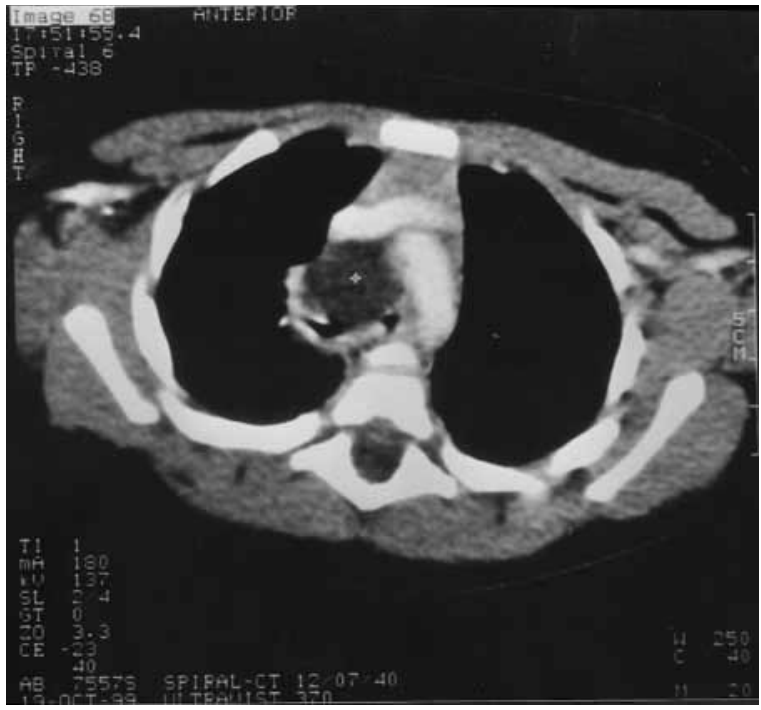

Figure 4. Computed tomographic scan without contrast medium showed a large homogeneous structure $(3.5 \times 2 \times 1.5 \mathrm{~cm})$ with fluid-equivalent signal intensity (Hounsfield units 10-20); there was no enhancement by contrast injection. The structure was localized in the middle mediastinum, on top of the heart, ventral to the tracheal bifurcation and dorsal to the right pulmonary artery. Compression of the trachea and main bronchi was evident.

$28 .^{2}$ The area presents a wide variety of tissues because of embryonic relationship of the branchial pouches, foregut primordium, pleuropericardial folds, and pharyngeal pouches. Various solid or cystic masses can be the result of the abnormal budding (eg, bronchogenic cysts, pericardial cysts, thymic cysts, and esophageal duplications). Bronchogenic cysts are the most common type of intrathoracic foregut cysts $(71 \%) .{ }^{4}$ They are mostly solitary and roughly spherical. A nonpatent stalk to one of the airways may exist.

The first successful surgical resection of bronchogenic cysts was reported in 1948 by Maier. ${ }^{5}$ Maier classified mediastinal bronchogenic cysts into 5 groups on the basis of their topography: paratracheal, carinal, hilar, paraesophageal, and miscellaneous (eg, in the diaphragm, chin, parasternal soft tissue, lower cervical areas, or scapular region). ${ }^{6}$

Histologically, bronchogenic cysts are usually thin walled, lined with columnar respiratory epithelium, and filled with protein-rich mucoid material. Seromucous glands (53\%), smooth muscle $(80 \%)$, and hyaline cartilage $(7 \%)$ are found. ${ }^{6}$

Bronchogenic cysts can be classified as mediastinal bronchogenic cysts $(86 \%)$ or bronchogenic cysts surrounded by lung parenchyma (14\%) (Table 1). The developmental stage of the budding is responsible for the location of the cysts.

Radiographically, the cyst appears to be a well-defined round mass. Calcification of the cyst wall is uncommon. Computed tomographic scan demonstrates a sharply demarcated, homogeneous structure. These masses are usually the density of water (lower attenuation), but they may be misinterpreted as solid masses when the internal contents are mucoid (high attenuation). Likewise, T1-weighted magnetic resonance imaging may demonstrate a high intensity because of protein-rich contents (compared 
with signal intensity between that of subcutaneous fat and muscle), or the magnetic resonance signal may be indicative of water. ${ }^{7,8}$ Enhancement after contrast injection is not described.

In our patient, radiographic definition of the cyst was not possible. All 3 chest x-ray films were unremarkable, and the diagnosis may have been delayed aas a consequence. The bronchoscopic examination gave the decisive pointer for the diagnosis and therapeutic strategy. The computed tomographic scan and the ultrasound examination were suggestive of a bronchogenic cyst.

Because of uncertainties in diagnosis and evolution, the removal of all cysts in children is recommended. The cysts do not regress spontaneously. On the contrary, they may fluctuate in size and tend to enlarge with bodily development or infection. The possibility for airway obstruction (as in our case), for infections, for ruptures, and even for malignant transformation exists and is unpredictable. ${ }^{2}$

Diagnosis and surgery were carried out at the Virchow-Clinic, Charité, Humboldt University, Berlin (Pediatric Radiology, Professor B. Stöver and U. Keske; Pediatric Cardiology, T. Boeckel; Pathology, Professor M. Vogel). We thank all participants.

\section{References}

1. Sharif N, Wiseman NW, Higgins M, Chernik V. Abnormal flowvolume loop leading to the diagnosis of bronchogenic cyst. Pediatr Pulmonol. 1999;27:218-20.

2. Ribet ME, Copin MC, Gosselin B. Bronchogenic cysts of the mediastinum. J Thorac Cardiovasc Surg. 1995;109:1003-10.

3. Myer CM, Cotton RT, Shot SR. The pediatric airway: an interdisciplinary approach. New York: JB Lippincott; 1995. p. 237, 240.

4. Michel JL, Revillon Y, Montupet P, Sauvat F, Sarnacki S, Sayegh N, et al. Thoracoscopic treatment of mediastinal cysts in children. J Pediatr Surg. 1998;33:1745-8.

5. Maier H. Bronchogenic cysts of the mediastinum. Ann Surg. 1948; 127:476-501.

6. Andryk JE, Kerschner JE, Hung RT, Aiken JJ, Conley SF. Mid-line cervical cleft with a bronchogenic cyst. Int $J$ Pediatr. Otorhinolaryngology 1999;47:261-4.

7. Lugo-Olivieri C, Schwarzman GJ, Beall DP, Lima JAC, Fishman EK. Intrapericardial bronchogenic cyst: assessment with magnetic resonance imaging and transesophageal echocardiography. Clin Imaging. 1999;23:81-4.

8. Kuhlman J, Fishman E, Wang K, et al. Mediastinal cysts: diagnosis by CT and needle aspiration. AJR Am J Roentgenol. 1988;150:75-8. 